
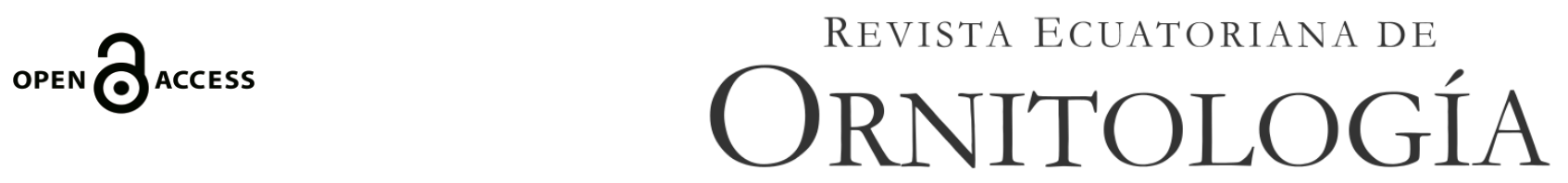

NOTA/NOTE

\title{
Nuevo registro de la Garza Cucharón Cochlearius cochlearius (Pelecaniformes: Ardeidae) en el occidente de Ecuador
}

\author{
Luis Carrasco \\ Fundación para la Conservación de los Andes Tropicales, Marcos Jofre Oe5-227 y Pasaje Esteban de la Rosa. Quito, Ecuador y Centro de \\ Investigación de la Biodiversidad y Cambio Climático, Universidad Tecnológica Indoamérica, Machala y Sabanilla. Quito, Ecuador. \\ luiscarrasco27@gmail.com \\ Editado por/Edited by: Esteban Guevara \\ Recibido/Received: 12 Septiembre 2018 Aceptado/Accepted: 9 Noviembre 2018 \\ Publicado en línea/Published online: 21 Noviembre 2019
}

New record of Boat-billed Heron Cochlearius cochlearius (Pelecaniformes: Ardeidae) in western Ecuador

\section{Resumen}

La Garza Cucharón Cochlearius cochlearius es una ave rara que habita en riberas de cuerpos de agua desde México hasta Argentina. En Ecuador es un ave rara y localista que se distribuye principalmente en la región amazónica. Al occidente existen registros históricos documentados en las provincias de Esmeraldas (1901) y Los Ríos (1931). Aquí se presenta una nueva observación, con evidencia fotográfica, de una pareja y un juvenil en el sector de la bananera Elba, provincia de Los Ríos, el 19 de septiembre de 2015. La presencia de un juvenil sugiere que la especie se está reproduciendo localmente y que podría ser residente en el área, pero sus hábitos nocturnos la hacen difícil de localizar. Este reporte constituye el redescubrimiento de la especie después de 84 años del último registro evidenciado de la especie en el occidente del Ecuador.

Palabras clave: Cochlearius cochlearius, Los Ríos, distribución.

\begin{abstract}
The Boat-billed Heron Cochlearius cochlearius is a rare bird that inhabits wetlands from Mexico to Argentina. In Ecuador, it is a rare and local species distributed primarily in the Amazonian lowlands. In the Pacific region of Ecuador, there are historical records from the provinces of Esmeraldas (1901) and Los Ríos (1931). Here, I report a new observation, with photographic evidence, of one pair and one juvenile from Bananera Elba, province of Los Ríos, on 19 September 2015. The presence of a juvenile suggests that the species is breeding locally and is resident in the area. The species might have remained overlooked due to its nocturnal habits. This report constitutes a rediscovery of the species 84 years after the last documented record in western Ecuador.
\end{abstract}

Keywords: Los Ríos, distribution, Cochlearius cochlearius.

La Garza Cucharón Cochlearius cochlearius tiene una amplia distribución en riberas de cuerpos de agua desde México hasta Argentina (Erize et al., 2006; Restall et al., 2006). En Ecuador, su rango de distribución incluye las tres regiones continentales (Occidente, Andes y Amazonía). La mayoría de registros proviene de la Amazonía, bajo los 400 m s.n.m. (Ridgely \& Greenfield, 2001; Ridgely \& Greenfield, 2006; Freile \& Restall, 2018). En los Andes existen solo dos registros de individuos errantes (Bahamonde-Vinueza et al., 2014), mientras que en el Occidente se conocen solamente tres especímenes con localidad cierta, dos especímenes con localidad imprecisa y un registro sin documentación (Fig. 1). El primer espécimen proviene de Salidero, provincia de Esmeraldas, colectado en 1901 y depositado en el Museo Americano de Historia Natural (AMNH 469471). Los otros dos fueron colectados en 1931 en Isla Silva, río Babahoyo, provincia de Los Ríos, y están depositados en el Museo Field de Chicago (FMNH 77354, 77355) (Ridgely \& Greenfield, 2001). Hay un registro no documentado de la provincia de Guayas, en 1982 (Man-Ging, 1982). Además, existe un espécimen en el Museo de Historia Natural Gustavo Orcés V. de la Escuela Politécnica Nacional (MEPN 894) cuyo único dato es "Occidente". Por último, hay dos registros históricos de juveniles colectados en las estribaciones occidentales, en la zona de Mindo, Río Blanco, en 1908 y 1909, pero no se tiene certeza de la validez de la localidad de colecta (Lönnberg \& Rendahl, 1922; Ridgely \& Greenfield, 2001; Bahamonde-Vinueza et al., 2014). 
Obtuve un nuevo registro de esta especie en la provincia de Los Ríos, cantón Pueblo Viejo, sector de la bananera Elba $(-1,692123,-79,587075,10$ m s.n.m., Fig. 1). En el lugar se realizó un inventario rápido de aves para conocer el aporte de los remanentes de vegetación para la conservación de aves en una matriz agrícola. Se implementaron ocho puntos de conteo, cuatro en vegetación boscosa intervenida y cuatro en vegetación a orillas de canales de agua. El muestreo efectivo se realizó en una visita de un día (19 de septiembre de 2015), y cada punto de conteo se recorrió a pie, por 20 min, para obtener registros visuales y auditivos (Bibby et al., 1998; Ralph et al., 2006). Según el mapa de ecosistemas del Ecuador (MAE, 2013), el lugar originalmente pertenecía al ecosistema Bosque semideciduo de tierras bajas de Jama-Zapotillo. Actualmente, existe un remanente de bosque degradado, con dominancia de árboles de samán (Samanea saman), rodeado de plantaciones extensas de banano.

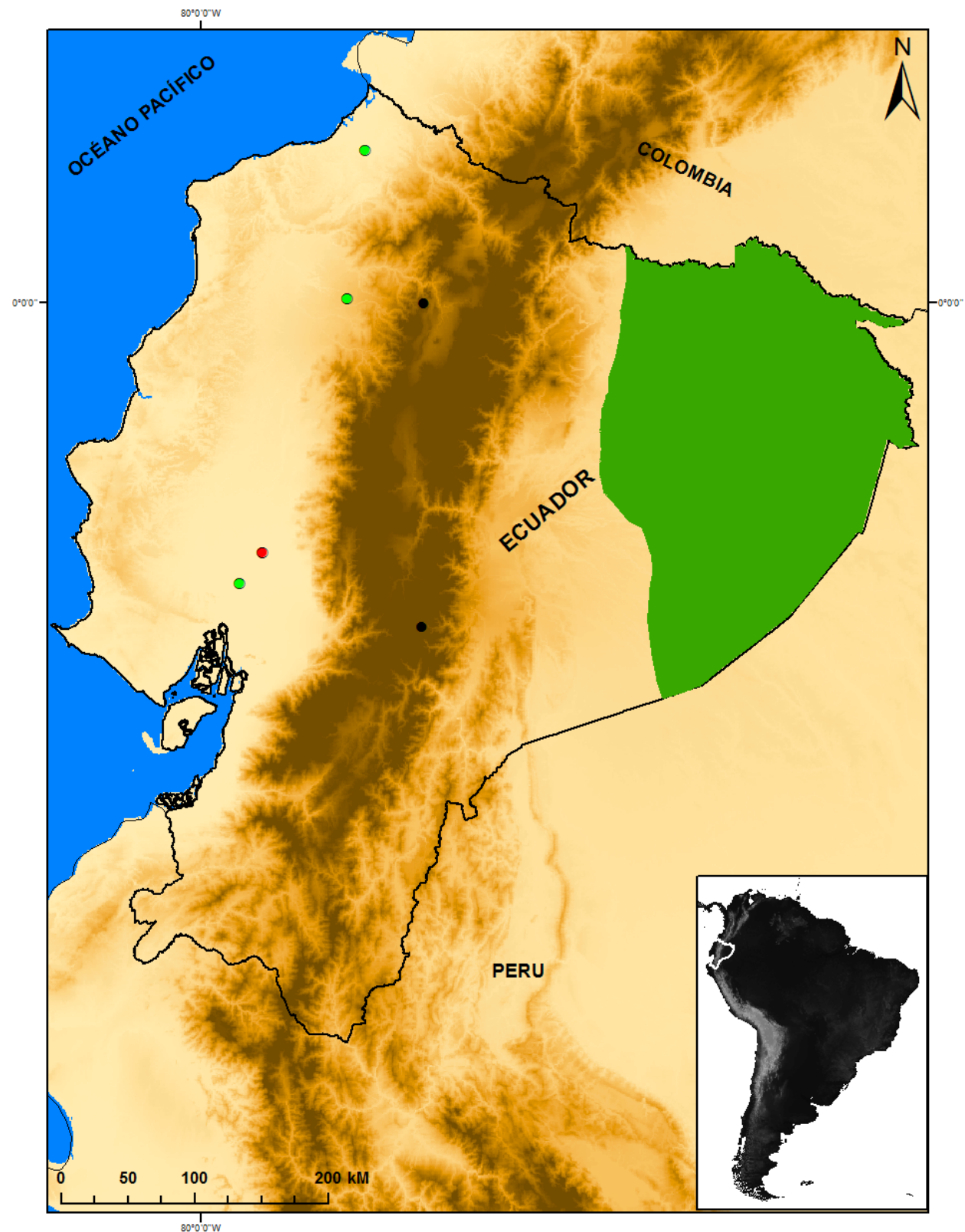

Figura 1: Distribución de Cochlearius cochlearius en Ecuador. En el occidente se presentan los registros históricos en verde: desde el norte, Salidero, Esmeraldas (1901); Río Blanco, Pichincha (1908, 1909); Isla Silva, Los Ríos (1931); en rojo el registro nuevo en Pueblo Viejo, Los Ríos (2015). En negro se presentan los registros en los Andes: desde el norte, Quito, Pichincha (2014); Isla Colay, Laguna de Atillo, Chimborazo (2004). En verde se muestra su distribución aproximada en la Amazonía ecuatoriana, adaptado de Ridgely \& Greenfield (2006).

Observé y fotografié tres individuos, dos adultos y un juvenil, de C. cochlearius en uno de los cuatro puntos de conteo ubicados en la vegetación a orillas de canales de agua (Fig. 2). Los adultos fueron identificados por su pico grande y ancho, ojos de gran tamaño y cabeza con frente blanca y penacho negro, mientras que el juvenil 
presentaba coloración mayormente marrón (Ridgely \& Greenfield, 2006; McMullan \& Navarrete, 2017; Freile \& Restall, 2018). Los individuos perchaban a una altura de $5 \mathrm{~m}$ en ramas de sotacaballo (Zygia longifolia). Estos árboles se encontraban en forma de hilera en la orilla del canal de agua, alcanzaban una estatura de $6 \mathrm{~m}$ y presentaban un follaje denso y numerosos frutos en forma de vainas alargadas que dificultaban la visualización hacia su interior.
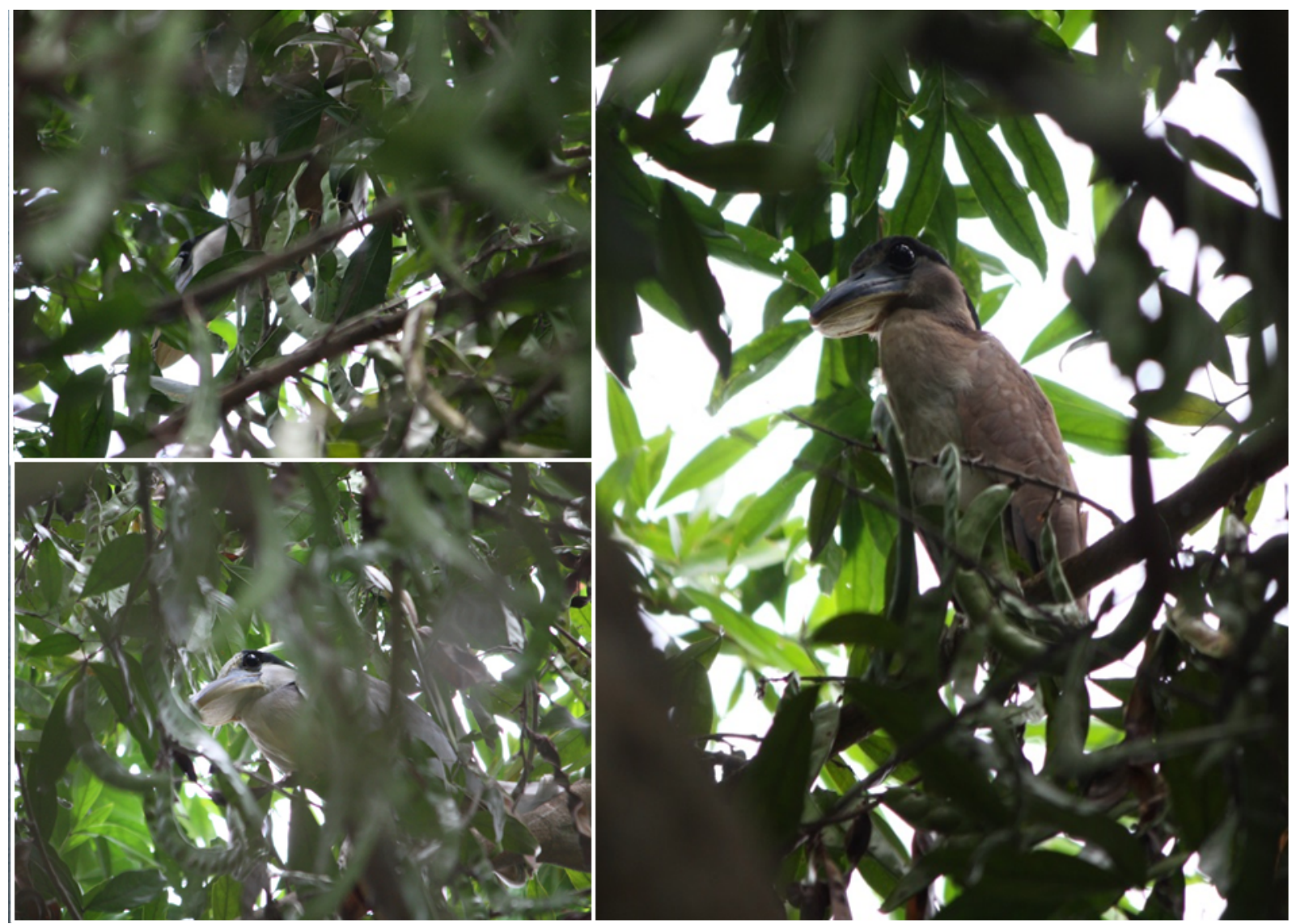

Figura 2: Dos individuos adultos y un juvenil de Garza Cucharón Cochlearius cochlearius perchados en árbol de sotacaballo (Zygia longifolia), en la bananera Elba, provincia de Los Ríos, 19 de septiembre de 2015.

El registro de una pareja con un juvenil sugiere reproducción local, que no se había reportado previamente en el occidente de Ecuador (Ridgely \& Greenfield, 2001), aunque los especímenes juveniles de "Río Blanco" sugerirían que existió actividad reproductiva. El registro reciente más cercano a esta nueva localidad proviene del embalse Tinajones, cerca de Chongoyape, departamento de Lambayeque, noroccidente de Perú (en 2005), donde se observó un individuo juvenil solitario que murió en poco tiempo, presumiblemente un vagabundo de las poblaciones amazónicas (Salazar et al., 2006). En el Pacífico colombiano, Ayerbe-Quiñones et al. (2008) reportaron un individuo colectado en el departamento de Cauca, en la zona costera e insular del Pacífico, aunque sin detalle de los datos de colección. Estos registros aislados sugieren que no existen poblaciones establecidas en el occidente de América del sur, por lo que sería poco probable que exista un flujo de individuos en la región. Por ello, se ha sugerido que los registros al occidente de los Andes comprenden individuos solitarios y vagabundos desde la Amazonía (Salazar et al., 2006; Bahamonde-Vinueza et al., 2014).

En México, en los meses inmediatos al anidamiento, generalmente en los manglares, los volantones se adentran hacia la vegetación cercana con follaje denso, haciéndose difícil su observación (Juárez \& Dickerman, 1972; Hernández \& Fernández, 1999). Consecuentemente, podemos inferir que la percha de los tres individuos aquí reportados en los árboles de follaje denso es parte de su estrategia de camuflaje para evitar a posibles depredadores. La percha diurna de C. cochlearius en árboles con follaje denso, ubicados en lugares alejados, y su hábito nocturno, podrían explicar que la especie haya pasado desapercibida en la región occidental del Ecuador. Este registro muestra la necesidad de mayor investigación ornitológica en la provincia de Los Ríos, incluso en zonas bajo fuerte influencia antrópica. 
A pesar de ser una especie rara, aunque fácil de identificar (Freile \& Restall, 2018), el último registro documentado en el occidente del Ecuador data de 1931. Por ello, se la ha reportado como posiblemente extirpada en esta región (Athanas \& Greenfield, 2016). El reporte de tres individuos que se presenta en esta nota constituye el primer registro documentado de la especie 84 años después de las últimas colectas. Esto pone en evidencia la importancia para la conservación de los escasos remanentes de vegetación natural en la provincia de Los Ríos. Es probable que el poco interés de investigación en Los Ríos se deba a la tala masiva de los bosques nativos, pues hasta el año 2016 solo quedaba el 1,3\% de bosque nativo original en esta provincia (MAE, 2017).

\section{AGRADECIMIENTOS}

Un agradecimiento a los propietarios de la bananera Elba por permitirnos el ingreso, a Jordan Karubian, Renata Durães, David Donoso, Elisa Bonaccorso, Juan Freile, Tatiana Santander y Boris Tinoco por la revisión del texto y sus acertados comentarios, a Rebeca Cruz por su asistencia en el campo y a Richard Quillupangui por su ayuda con la elaboración del mapa.

\section{REFERENCIAS}

Athanas, N., \& Greenfield, P. (2016). Birds of western Ecuador: a photographic guide. Princeton, New Jersey: Princeton University Press.

Ayerbe-Quiñones, F., López-Ordóñez, J. P., González-Rojas, M. F., Estela, F. A., Ramírez-Burbano, M. B., Sandoval-Sierra, J. V., \& Gómez-Bernal, L. G. (2008). Aves del departamento del Cauca-Colombia. Biota Colombiana, 9(1), 77-132. DOI: https://doi.org/10.21068/bc.v9i1.211

Bahamonde-Vinueza D., Cadena-Ortiz, H., Cajas-Bermeo, C. \& Bonaccorso, E. (2014). Unusual records of Cochlearius cochlearius (Linnaeus, 1766) (Aves: Ardeidae) in the Andes of Ecuador. Check List, 10(3), 687688. DOI: http://dx.doi.org/10.15560/10.3.687

Bibby, C., Jones, M., \& Marsden, S. (1998). Expedition field techniques, bird surveys. Londres, UK: Royal Geographical Society.

Erize, F., Rodríguez, J. R., \& Rumboll, M. (2006). Birds of South America: non-passerines: rheas to woodpeckers. New Jersey, New Jersey: Princeton University Press.

Freile, J. F. \& Restall, R. (2018). Birds of Ecuador. Londres, UK: Helm Field Guides.

Hernández, S., \& Fernández, G. (1999). Reproducción de Cochlearius cochlearius (garza cucharón) y Butorides virescens (garza verde) en la Manzanilla, Jalisco, México. Ciencias Marinas, 25(2), 277-291.

Juárez, C., \& Dickerman, R. (1972). Nestling development of Boat-Billed Herons (Cochlearius cochlearius) at San Blas, Nayarit, Mexico. Wilson Bulletin, 84(4), 456-68. URL: https://sora.unm.edu/sites/default/files/journals/wilson/v084n04/p0456-p0468.pdf

Lönnberg, E. \& Rendahl, H. (1922). A contribution to the ornithology of Ecuador. Arkiv föh Zoologi 14(25), 187. URL: https://ia800207.us.archive.org/35/items/arkivfrzoologi14kung/arkivfrzoologi14kung.pdf

Man-Ging, F. (1982, Diciembre 6). eBird checklist: https://ebird.org/view/checklist/S30974640. eBird: an online database of bird distribution and abundance. Ithaca, New York. URL: http://www.ebird.org

McMullan M., \& Navarrete L. (2017). Fieldbook of the birds of Ecuador including the Galapagos Islands and common mammals. Quito: Ratty Ediciones.

Ministerio del Ambiente del Ecuador. (2013). Sistema de clasificación de los ecosistemas del Ecuador continental. Quito: Subsecretaría de Patrimonio Natural, Ministerio del Ambiente.

Ministerio del Ambiente del Ecuador. (2017). Deforestación del Ecuador continental, periodo 2014-2016. Quito: Ministerio del Ambiente del Ecuador. 
Ralph, C. J., Geupel, G. R., Pyle, P., Martin, T. E., DeSante, D. F., \& Milá, B. (1996) Manual de métodos de campo para el monitoreo de aves terrestres. Albany, CA: Pacific Southwest Research Station, Forest Service, U.S. Department of Agriculture.

Restall, R., Rodner, C., \& Lentino, M. (2006). Birds of northern South America. An identification guide. Londres, UK: Helm Field Guides.

Ridgely, R. S., \& Greenfield, P.J. (2001). The birds of Ecuador. Ithaca, New York: Cornell University Press.

Ridgely, R. S., \& Greenfield, P.J. (2006). Aves del Ecuador. Quito: Academia de Ciencias de Philadelphia \& Fundación Jocotoco.

Salazar, T., Plenge, H. \& Williams, R. S. R. (2006). Boat-billed Heron Cochlearius cochlearius in Lambayeque, western Peru. Cotinga, 26, 83. 